\title{
Phase-Shifting the Fruit Fly Clock without Cryptochrome
}

\author{
Christa Kistenpfennig, ${ }^{*,+}$ Jay Hirsh, ${ }^{\ddagger}$ Taishi Yoshii, ${ }^{*,+,, 1}$ and Charlotte Helfrich-Förster ${ }^{*,+, 1}$ \\ *Institute of Zoology, University of Regensburg, Regensburg, Germany, \\ ${ }^{+}$Neurobiology and Genetics, Theodor-Boveri Institute, Biocenter, \\ University of Würzburg, Am Hubland, Würzburg, Germany, \\ ${ }^{\ddagger}$ Department of Biology, University of Virginia, Charlottesville, VA, USA, and \\ ${ }^{\S}$ Graduate School of Natural Science and Technology, Okayama University, Okayama, Japan
}

\begin{abstract}
The blue light photopigment cryptochrome (CRY) is thought to be the main circadian photoreceptor of Drosophila melanogaster. Nevertheless, entrainment to light-dark cycles is possible without functional CRY. Here, we monitored phase response curves of $c r y^{01}$ mutants and control flies to 1-hour 1000-lux light pulses. We found that $c r y^{01}$ mutants phase-shift their activity rhythm in the subjective early morning and late evening, although with reduced magnitude. This phase-shifting capability is sufficient for the slowed entrainment of the mutants, indicating that the eyes contribute to the clock's light sensitivity around dawn and dusk. With longer light pulses ( 3 hours and 6 hours), wild-type flies show greatly enhanced magnitude of phase shift, but CRY-less flies seem impaired in the ability to integrate duration of the light pulse in a wild-type manner: Only 6-hour light pulses at circadian time 21 significantly increased the magnitude of phase advances in $c r y^{01}$ mutants. At circadian time 15, the mutants exhibited phase advances instead of the expected delays. These complex results are discussed.
\end{abstract}

Key words cryptochrome, light pulses, locomotor activity, Drosophila melanogaster

The clock of the fruit fly Drosophila melanogaster is extremely light sensitive to entrainment, using 12: 12-hour light-dark (LD) cycles of very dim light (Stanewsky et al., 1998; Ohata et al., 1998; HelfrichFörster et al., 2001; Bachleitner et al., 2007; Hirsh et al., 2010). Adult flies re-entrain to 8-hour shifts of bright LD cycles within 1 or 2 days (Emery et al., $2000 \mathrm{~b}$ ). In contrast, mammals need a minimum of 1 week to re-entrain to such phase shifts (Aschoff et al., 1975). The fly possesses many photoreceptors, but the blue light photopigment cryptochrome (CRY) is regarded as the main photoreceptor responsible for the high light sensitivity of the fly's clock (Emery et al., 1998, 2000a, 2000b). CRY is expressed in the majority of clock neurons, where it interacts with the clock protein Timeless (TIM), provoking its lightdependent degradation (Benito et al., 2008; Yoshii et al., 2008). Without functional CRY, TIM is not degraded upon exposure to constant light (LL). As a consequence, $\mathrm{cry}^{b}$ mutants that carry a point mutation in the flavin binding region of cryptochrome as well as cry-null (cry and cry $\left.{ }^{o u t}\right)$ mutants remain rhythmic under LL even at intensities above 1000 lux (Emery et al., 2000a; Yoshii et al., 2004; Rieger et al.,

1. To whom all correspondence should be addressed: Charlotte Helfrich-Förster and Taishi Yoshii, Lehrstuhl für Genetik und Neurobiologie, Universität Würzburg, Biozentrum, Am Hubland, 97074 Würzburg, Germany; e-mail: charlotte. foerster@biozentrum.uni-wuerzburg.de and taishi.yoshii@biozentrum.uni-wuerzburg.de.

JOURNAL OF BIOLOGICAL RHYTHMS, Vol. 27 No. 2, April 2012 117-125

DOI: $10.1177 / 0748730411434390$

(C) 2012 The Author(s) 
2006; Dolezelova et al., 2007), whereas wild-type flies and mutants without functional eyes become arrhythmic at intensities beyond 10 lux (Konopka et al., 1989; Helfrich-Förster et al., 2001). Furthermore, $c^{r} y^{b}$ mutants are not able to shift their activity rhythms in response to short (10-minute) light pulses (Stanewsky et al., 1998).

Despite the importance of CRY for circadian photoreception, cry mutants can entrain well to LD cycles (Stanewsky et al., 1998), although they require longer time to re-entrain to 8-hour shifted LD cycles (Emery et al., 2000b). Similar slow responses to 8-hour phase shifts are rather common for mammalian species that have no photoreceptive pigment in their clock neurons. In mammals, light is exclusively perceived by the eyes and is mediated to the clock in the suprachiasmatic nuclei $(\mathrm{SCN})$ via glutamate and PACAP through regular synapses onto retinorecipient clock neurons in the ventrolateral SCN core (Morin and Allen, 2006). The clock neurons of D. melanogaster also receive light input from photoreceptor cells of the compound eyes, the Hofbauer-Buchner eyelets ( $\mathrm{H}-\mathrm{B}$ eyelets), and perhaps other unidentified interneurons (Helfrich-Förster et al., 2001; Rieger et al., 2003; Veleri et al., 2003; Veleri et al., 2007), although direct synaptic connections have only been shown between photoreceptor cells and clock neurons of larvae so far (Wegener et al., 2004). This eye-mediated light input is probably sufficient for a normal entrainment of the activity rhythm that largely resembles that of mammals. If true, CRY-deficient fruit flies should show a low-amplitude phase response curve (PRC).

To determine if this is true, we characterized the phase-shifting capabilities of CRY-less flies $\left(c r y^{01}\right.$ mutants) by monitoring a PRC to light pulses of 1-hour duration. We found that $c r y^{01}$ mutants are able to phase-shift their clock, although the magnitude of phase shifts was reduced to approximately $25 \%$ of control flies. Thus, our results can explain the re-entrainment characteristics of CRY-deficient flies.

\section{MATERIALS AND METHODS}

\section{Fly Strains}

To exclude any residual function of CRY, we used mutants that lack CRY completely $\left(c r y^{01}\right)$ (Dolezelova et al., 2007) instead of $c r y^{b}$ mutants that show just one amino acid change in the CRY flavin binding domain that is crucial for light reception (Stanewsky et al., 1998). $c r y^{01}$ flies are knockout mutants generated from $w^{1118}$ flies by homologous recombination, in which the entire coding sequence of the $\mathrm{cry}^{+}$allele was replaced by mini-white ${ }^{+}$(Dolezelova et al., 2007). In addition, $c r y^{01}$ was outcrossed to the $w^{1118}$ Bloomington strain no. 6326 (Dolezelova et al., 2007). This $w^{1118}$ strain was used as a control strain in the present experiments, so that mutant $\left(w^{1118 ;} ; ; r y^{01}\right)$ and control flies $\left(w^{1118}\right)$ had exactly the same genetic background except for the cry and the mini-white ${ }^{+}$gene. Both strains carried the timeless allele s-tim and the wild-type jetlag gene (jet ${ }^{+}$) (Dolezelova et al., 2007) and should therefore have a molecular clock of similar light sensitivity (Peschel et al., 2006). For simplicity, we will use "cry ${ }^{01 "}$ for " $w^{1118}$ ";cry $y^{01 "}$ and "control" for the " $w^{1118 "}$ strain throughout the article.

The flies were reared under LD 12:12 cycles on Drosophila medium (0.8\% agar, $2.2 \%$ sugar-beet syrup, $8.0 \%$ malt extract, $1.8 \%$ yeast, $1.0 \%$ soy flour, $8.0 \%$ corn flour, and $0.3 \%$ hydroxybenzoic acid) at either $20{ }^{\circ} \mathrm{C}$ or $25^{\circ} \mathrm{C}$. Only male flies at an age of 3 to 6 days were taken for the experiments.

\section{Recording the Locomotor Activity of Flies}

Locomotor activity of individual male flies was recorded photoelectrically as described previously (Helfrich-Förster, 1998; Rieger et al., 2007). Briefly, the flies were confined to photometer cuvettes that were placed with one end in an infrared light beam. On the opposite end, they had access to water and sugar. Activity was monitored during consecutive 1-minute intervals. Light was provided by white LEDs (Lumitronix LED-Technik $\mathrm{GmbH}$, Jungingen, Germany). The recording units were placed in a temperature-controlled room or an incubator (I-36NL, Percival Scientific Inc., Perry, IA). The temperature was kept constant at $20^{\circ} \mathrm{C}$ throughout all experiments.

For determining the shifting behavior of the flies, these were monitored under LD cycles (12:12) for 7 days either at 100,1000, or 10,000 lux $\left(19 \mu \mathrm{W} / \mathrm{cm}^{2}\right.$, $150 \mu \mathrm{W} / \mathrm{cm}^{2}$, or $1300 \mu \mathrm{W} / \mathrm{cm}^{2}$, respectively), and then, the LD cycle was phase-delayed by 8 hours. Intensity was controlled with neutral density filters and by changing the voltage/current.

For monitoring PRCs, the flies were entrained to LD cycles (12:12) for 5 days (100 lux or $19 \mu \mathrm{W} / \mathrm{cm} 2$ ) and then transferred to DD and recorded for at least a further 10 days under DD. One group consisting of 59 control and $27 \mathrm{cry} \mathrm{C}^{01}$ flies was recorded without any disturbance to assess mean period and initial phase of the free-running rhythms (Fig. 1 and below). The other flies received a light pulse of 1-hour duration and a light intensity of 1000 lux $(150 \mu \mathrm{W} / \mathrm{cm} 2)$ during the first day of DD at different circadian times 
Table 1. Phase responses of control and $c r y^{01}$ flies to a 60-minute light pulse at different times of day.

\begin{tabular}{|c|c|c|c|c|c|c|c|c|c|c|c|c|}
\hline $\mathrm{CT}$ & 01 & 03 & 05 & 07 & 09 & 11 & 13 & 15 & 17 & 19 & 21 & 23 \\
\hline \multicolumn{13}{|c|}{ Control } \\
\hline Phase shift, $\mathrm{h}$ & $\begin{array}{c}1.35 \\
\pm 0.44 \\
\end{array}$ & $\begin{array}{c}0.57 \\
\pm 0.25 \\
\end{array}$ & $\begin{array}{c}0.52 \\
\pm 0.18 \\
\end{array}$ & $\begin{array}{l}-0.11 \\
\pm 0.22 \\
\end{array}$ & $\begin{array}{c}0.15 \\
\pm 0.13 \\
\end{array}$ & $\begin{array}{c}0.18 \\
\pm 0.25 \\
\end{array}$ & $\begin{array}{r}-0.45 \\
\pm 0.33 \\
\end{array}$ & $\begin{array}{r}-4.05 \\
\pm 0.18^{\mathrm{a}} \\
\end{array}$ & $\begin{array}{c}-3.48 \\
\pm 0.45^{\mathrm{a}} \\
\end{array}$ & $\begin{array}{c}1.17 \\
\pm 0.30^{\mathrm{a}}\end{array}$ & $\begin{array}{c}2.75 \\
\pm 0.20^{\mathrm{a}} \\
\end{array}$ & $\begin{array}{c}1.34 \\
\pm 0.18^{\mathrm{a}} \\
\end{array}$ \\
\hline \multicolumn{13}{|c|}{$c r y^{01}$} \\
\hline Phase shift, $\mathrm{h}$ & $\begin{array}{c}0.31 \\
\pm 0.19\end{array}$ & $\begin{array}{c}0.31 \\
\pm 0.25\end{array}$ & $\begin{array}{c}0.38 \\
\pm 0.26\end{array}$ & $\begin{array}{l}-0.14 \\
\pm 0.21\end{array}$ & $\begin{array}{c}0.03 \\
\pm 0.14\end{array}$ & $\begin{array}{l}-0.57 \\
\pm 0.19\end{array}$ & $\begin{array}{l}-0.92 \\
\pm 0.28\end{array}$ & $\begin{array}{c}-0.97 \\
\pm 0.21^{\mathrm{a}}\end{array}$ & $\begin{array}{l}-0.11 \\
\pm 0.15\end{array}$ & $\begin{array}{l}-0.27 \\
\pm 0.17\end{array}$ & $\begin{array}{c}1.05 \\
\pm 0.27^{\mathrm{a}}\end{array}$ & $\begin{array}{c}1.05 \\
\pm 0.21^{\mathrm{a}}\end{array}$ \\
\hline
\end{tabular}

Values are shown as mean \pm SEM.

a. The phase shift was statistically significant compared with nonpulsed flies.

Table 2. Phase responses of control and $c r y^{01}$ flies to light pulses with various durations at CT15 and CT21.

\begin{tabular}{|c|c|c|c|c|c|c|c|c|}
\hline \multirow{2}{*}{\begin{tabular}{|l} 
Duration \\
$\mathrm{CT}$
\end{tabular}} & \multicolumn{2}{|c|}{$15 \mathrm{~min}$} & \multicolumn{2}{|c|}{$60 \mathrm{~min}$} & \multicolumn{2}{|c|}{$180 \mathrm{~min}$} & \multicolumn{2}{|c|}{$360 \mathrm{~min}$} \\
\hline & 15 & 21 & 15 & 21 & 15 & 21 & 15 & 21 \\
\hline \multicolumn{9}{|c|}{ Control } \\
\hline Phase shift, $h$ & $\begin{array}{c}-3.91 \\
\pm 0.22^{\mathrm{a}} \\
\end{array}$ & $\begin{array}{r}1.91 \\
\pm 0.28^{\mathrm{a}} \\
\end{array}$ & $\begin{array}{r}-4.05 \\
\pm 0.18^{\mathrm{a}} \\
\end{array}$ & $\begin{array}{c}2.75 \\
\pm 0.20^{\mathrm{a}} \\
\end{array}$ & $\begin{array}{c}-5.27 \\
\pm 0.23^{\mathrm{a}} \\
\end{array}$ & $\begin{array}{c}2.98 \\
\pm 0.28^{\mathrm{a}} \\
\end{array}$ & $\begin{array}{l}-10.73 \\
\pm 0.36^{\mathrm{a}} \\
\end{array}$ & $\begin{array}{c}5.73 \\
\pm 0.32^{\mathrm{a}} \\
\end{array}$ \\
\hline \multicolumn{9}{|c|}{ cry ${ }^{01}$} \\
\hline Phase shift, $h$ & $\begin{array}{c}-1.06 \\
\pm 0.28^{\mathrm{a}}\end{array}$ & $\begin{array}{c}0.40 \\
\pm 0.26\end{array}$ & $\begin{array}{c}-0.97 \\
\pm 0.21^{\mathrm{a}}\end{array}$ & $\begin{array}{c}1.05 \\
\pm 0.27^{\mathrm{a}}\end{array}$ & $\begin{array}{l}-1.37 \\
\pm 0.26^{\mathrm{a}}\end{array}$ & $\begin{array}{c}0.46 \\
\pm 0.19\end{array}$ & $\begin{array}{c}0.86 \\
\pm 0.19^{\mathrm{a}}\end{array}$ & $\begin{array}{c}1.69 \\
\pm 0.22^{\mathrm{a}}\end{array}$ \\
\hline
\end{tabular}

Values are shown as mean \pm SEM.

a. The phase shift was statistically significant compared with nonpulsed flies.

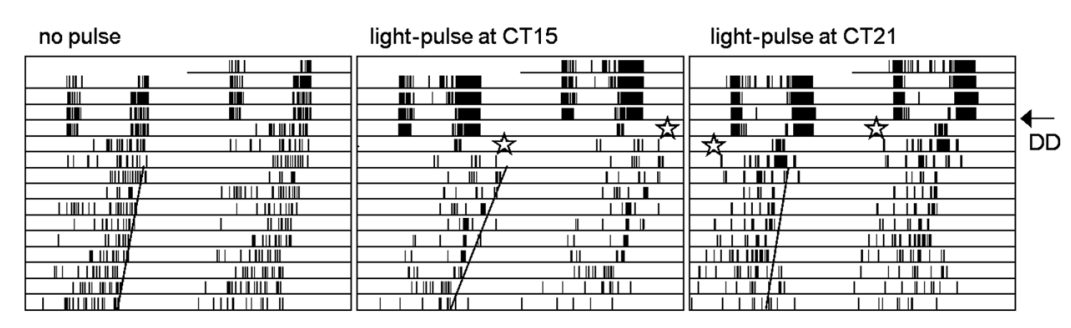

Figure 1. Method of administering light pulses and determining consecutive phase shifts in an anchored phase response curve. The light pulses (indicated by stars) were given either at CT15 or CT21 during the first day after the flies were released from 12:12 LD cycles. A line was drawn through the offset of the free-running activity and extrapolated back to determine the phase shift in comparison to unpulsed controls (detailed description in "Materials and Methods").

(CT1 to CT23 with 2-hour intervals). The given CT indicated the beginning of the 1-hour light pulse. CT0 was defined as the subjective beginning of the day and CT12 as the subjective beginning of the night. Thus, CT0 to CT24 is the duration of one endogenous cycle (period, $\tau$ ). The actual CT of the light pulse was calculated by multiplying the real hour by $24 \mathrm{~h} / \tau$ for each individual fly (Johnson, 1992). Similarly, the phase shifts were indicated as circadian hours (actual hours were multiplied by $24 \mathrm{~h} / \tau)$. PRCs were calculated for control flies and $c r y^{01}$ mutants as indicated under "Data Analysis".

To determine the dose response characteristics of phase shifts in respect to light pulse duration, we administered light pulses of the same intensity (1000 lux) for 15, 60, 180, and 360 minutes at either CT15 or
CT21 and, in a second experiment, 60-minute light pulses of 10,000 lux.

\section{Data Analysis}

The raw data of individual flies were displayed as actograms using the program El Temps (v. 1.228, Antoni Diez-Noguera; http:// www.el-temps.com/). The time needed for resynchronization to an 8-hour shift of the LD cycle was determined in each single fly by one experienced person who was blind to the genotype and the irradiance. Average values were calculated for the 2 genotypes at the 3 irradiances, and averaged actograms were plotted to visualize the phase-shifting behavior.

For monitoring the responses to the light pulses, the phase of the rhythms was determined by the offset of the evening activity because this was more stable than the onset and the peak of activity under free-running conditions. First, we determined the activity offset of flies that had not received any light pulse on the first day in DD (59 control and $27 c r y^{01}$ mutant flies) and calculated average phases for both genotypes. Those values were used as reference phases for the light-pulsed flies. To obtain the phase shift values for individual light-pulsed flies, their actual activity offset was determined on the actogram 


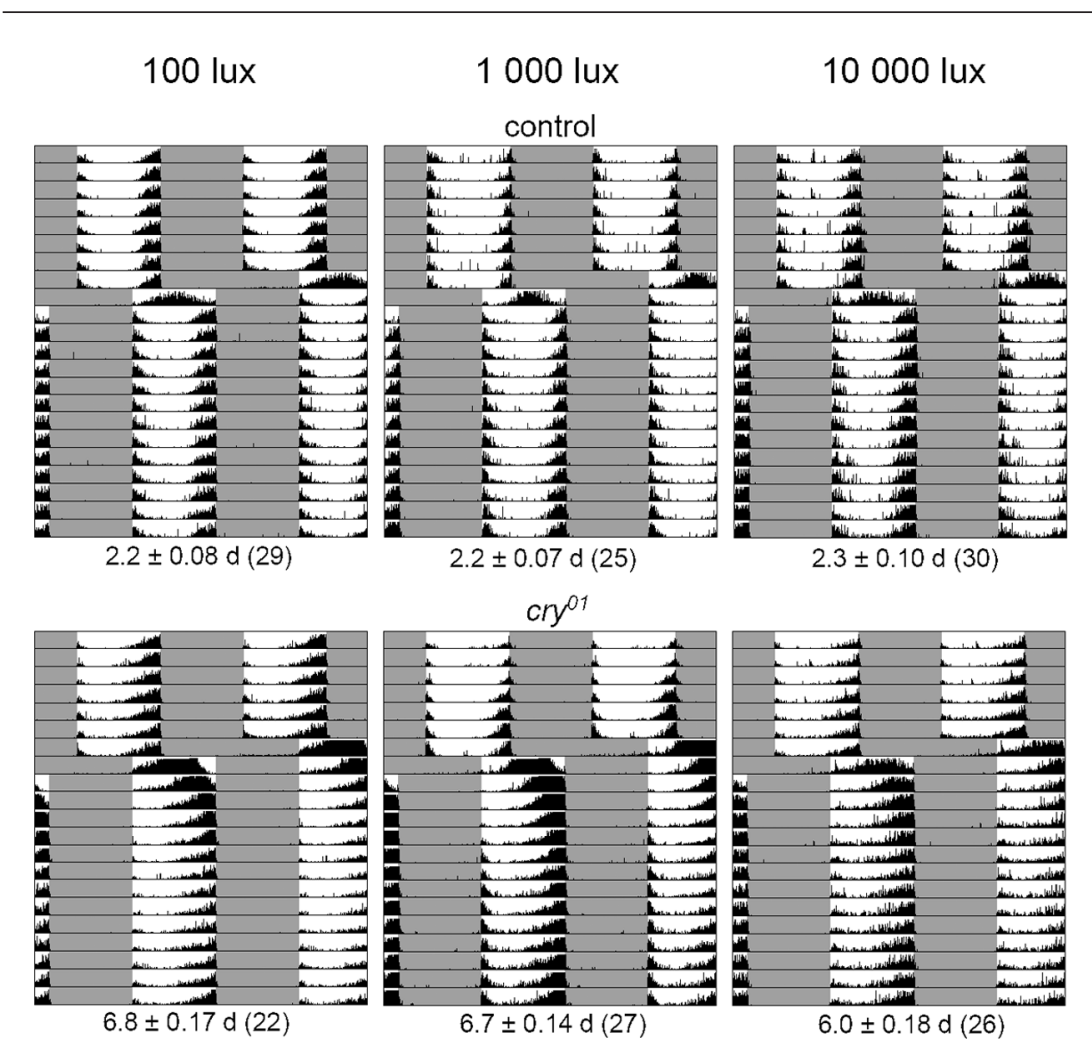

Figure 2. Average actograms of control and $c r y^{01}$ flies that were subjected to a phase delay of a 12:12 LD cycle by 8 hours (at 3 different light intensities). Below the average actograms, the number of days is given ( \pm SEM) that the flies needed to re-entrain as well as the number of tested flies (in parentheses). Controls shifted their activity quickly and were completely adapted to the new light schedule on the second day after the shift regardless of the light intensity during the day (Kruskal-Wallis 1-way analysis showed that re-entrainment did not depend on irradiance: $F_{2,92}=4.16, p=$ 0.125). $c r y^{01}$ mutants needed 6 to 7 days until they reached their original phase relation to the LD cycle, meaning that they shifted 1.3 hours per day at maximum. The phaseshifting capabilities between control flies and $\mathrm{cry}^{01}$ mutants were significantly different (Kruskal-Wallis 1-way analysis at all irradiances: $p=<0.00001)$. Furthermore, in $c r y^{01}$ mutants, the speed of re-entrainment was faster at 10,000 lux than at 100 and 1000 lux (Kruskal-Wallis 1-way analysis revealed the re-entrainment depended on irradiance: $F_{2,73}=12.85, p=0.002$; the Wilcoxon post hoc test showed that re-entrainment was significantly faster at 10,000 lux as compared to the 2 lower irradiances: $p=0.014$ ). and enabled a statistical comparison of the phase shift magnitude within and between the strains.

\section{Statistics}

The phase-shifting capabilities of controls and mutants to the 8-hour shift of the LD cycle were analyzed by the Kruskal-Wallis 1-way analysis followed by a Wilcoxon post hoc test (Systat 11, SPSS, Chicago, IL). Phase shifts after the light pulses were tested for a significant influence of time and genotype or duration of illumination and genotype using a 2-way ANOVA (Systat 11, SPSS). Few data sets were not normally distributed, as revealed by the KolmogorovSmirnov 1-sample test (Fig. 3). In these sets, $p$ was adjusted according to Glaser (1978) by multiplication by 2 . Values were regarded as significantly different at $p<0.05$.

\section{RESULTS}

Re-entrainment experiments to 8-hour LD cycle delays showed that control flies re-entrain within approximately 2 days and this speed cannot be enhanced further by higher irradiances (Fig. 2). In contrast, $c r y^{01}$ mutants needed 6 to 7 days to re-entrain, and the time to re-entrainment was reduced by 0.8 days when irradiance was increased by drawing a line through all activity offsets and extrapolating it back to the day the phase shift occurred (Fig. 1). The determined activity offset was then subtracted by the calculated reference phase, and the conversion into circadian hours was done (see above). The calculated phase shifts of all individual flies were plotted against the CT of the light pulse in a scatter plot. Because the periods of control and $c r y^{01}$ flies were not significantly different and close to 24 hours $\left({ }^{t} \mathrm{cry}{ }^{01}=23.79 \pm 0.05 \mathrm{~h} ;{ }^{t}\right.$ control $=23.86 \pm 0.06$ h), we plotted the PRC also on the basis of real time (without calculating the individual CTs). This method allowed the calculation of average phase shifts and standard errors of the mean (SEM) for each time point from 100 to 10,000 lux (Fig. 2). The phase-shifting behavior of $\mathrm{cry}^{01}$ mutants was very similar to that reported previously (Emery et al., 2000b); but in contrast to previous reports, we did not see any lights-on anticipation of morning activity. The latter can be explained by our recording system that misses small actions of the flies, such as movements between water and sugar, because the infrared light beam is on the opposite end of the cuvette (see Fig. 1 in Helfrich-Förster [1998]). If we monitor the activity of the flies with commercial Drosophila Activity Monitors (DAM, Trikinetics Inc., Waltham, MA), we see this morning anticipation (Yoshii and Helfrich-Förster, unpublished observations). 

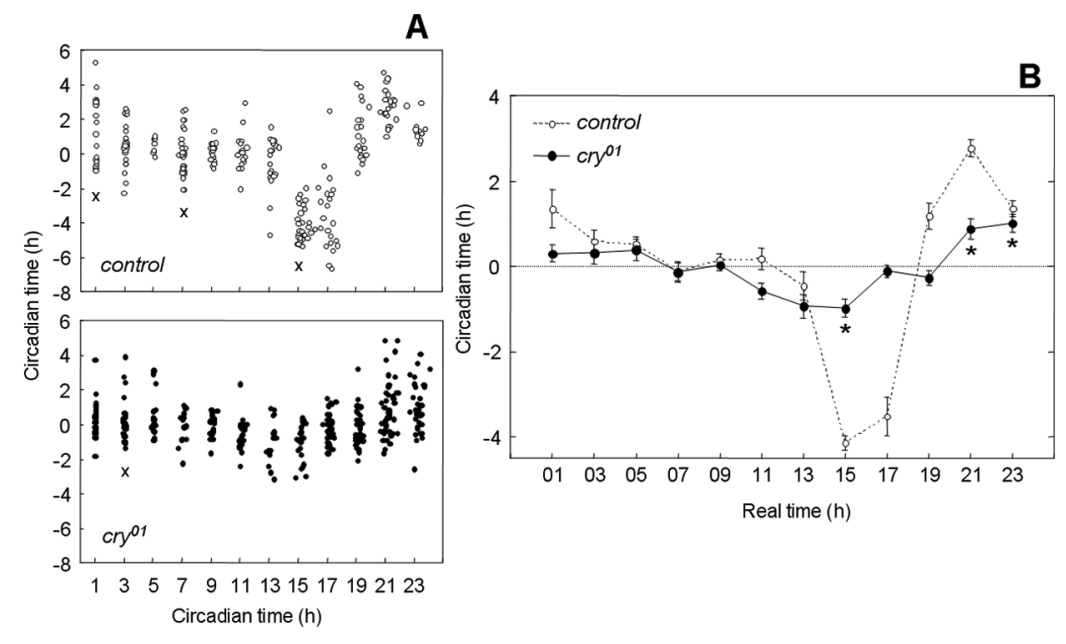

Figure 3. Phase response curves for control flies and $c r y^{01}$ mutants plotted in circadian time (CT) (A) and in real time (B). Flies were pulsed for 1 hour with white light (1000 lux) during the first subjective day of DD at the times indicated on the abscissa. Phase changes were calculated by comparing behavioral offsets of light-pulsed flies to the behavior of flies that did not receive a pulse. Phase delays and advances are plotted in circadian hours as negative and positive values, respectively. (A) The phase shifts of all light-pulsed individuals are shown as dots in CT. Crosses indicate the data sets that were not normally distributed. (B) Mean phase shifts $( \pm$ SEM) are calculated out of the individual phase shifts of all flies pulsed at the same real time (shown as dots in A). Asterisks indicate the phase advances/delays in $c r y^{01}$ mutants that were significantly different from unpulsed flies. ANOVA revealed that the phase shifts were highly dependent on time in both strains (control: $F_{11,238}=54.74, p<0.001 ; c r y^{01}$ mutants: $F_{11,320}=8.91, p<0.001$ ) and that they depended additionally significantly on the strain $\left(F_{11,558}=24.02, p<0.001\right)$.

Next, we tested the dependence of phase shift magnitude on length of the light pulse, varying pulse lengths between 15 and $360 \mathrm{~min}-$ utes. The light pulses were administered at the most sensitive parts of the clock in the delay (CT15) and advance (CT21) zones. After light pulses of 15 minutes, both strains showed significant phase delays, and control flies showed additionally significant phase advances (Fig. 4A). After longer light pulses, significant delays and advances were present in both strains, but $c r y^{01}$ mutants clearly behaved differently from control flies: Whereas delays and advances of controls increased significantly with increasing light pulse duration, this was not the case in $c r y^{01}$ mutants until a pulse duration of 180 minutes (3 hours). But when light pulse duration was increased to 6 hours, a significant change occurred: the light pulses at both time points provoked phase advances, and at CT21, these were slightly but significantly larger than the ones provoked by

Experiments giving entrained flies a 1-hour light pulse during the first day in DD revealed that $c r y^{01}$ mutants and control flies phase-shifted their activity, showing delays in the early night and advances in the late night and a dead zone in the middle of the subjective day. This pattern is evident in the scatter plot (Fig. $3 \mathrm{~A})$ and in the averaged PRC (Fig. 3B). ANOVA revealed that the phase shifts were highly dependent on time in both strains and that they depended additionally significantly on the strain. Control flies showed phase delays of up to approximately 4 hours and phase advances of approximately 2.5 hours, whereas $c r y^{01}$ mutants showed reduced phase changes of approximately 1 hour for both advances and delays (Fig. 3B). In both strains, maximal phase delays occurred at approximately CT15 and maximal phase advances at approximately CT21, but the shape of the PRC was different at its transition region: the control flies showed the expected rapid transition between delays and advances, but $\mathrm{cry}^{01}$ revealed a second small "dead zone" between the switch. As a consequence, the phase advance started later in $c r y^{01}$ mutants at CT21 than in the control flies at CT19. the shorter light pulses (Fig. 4A).

Next, we tested whether 1-hour light pulses of higher intensity could provoke larger phase shifts by light-pulsing control and $c r y^{01}$ mutants with 10,000 lux at CT15 or CT21. After this high intensity pulse, the majority of flies became inactive, especially after the CT21 pulse. At CT21, the small fraction of active flies phase-advanced their activity as expected, and there was a tendency to increase magnitude as compared to 1000-lux light pulses in $c r y^{01}$ mutants but not in control flies (Fig. 4B). Indeed, at 10,000 lux and CT21, the phase advances of $c r y^{01}$ mutants were not significantly different from the ones of control flies (ANOVA: $F_{1,7}=0.17, p=0.70$ ). At CT15, cry ${ }^{01}$ mutants did not phase-shift at all, whereas control flies showed no further increase in phase delays as compared to 1000 lux (Fig. 4B).

Our results demonstrate that the phase-shifting capability of wild-type but not of $c r y^{01}$ mutants can increase to extremely large values when time of the pulse is extended to 6 hours, indicating that a CRY-dependent mechanism must exist to allow large magnitude phase shifts from these long light pulses. 


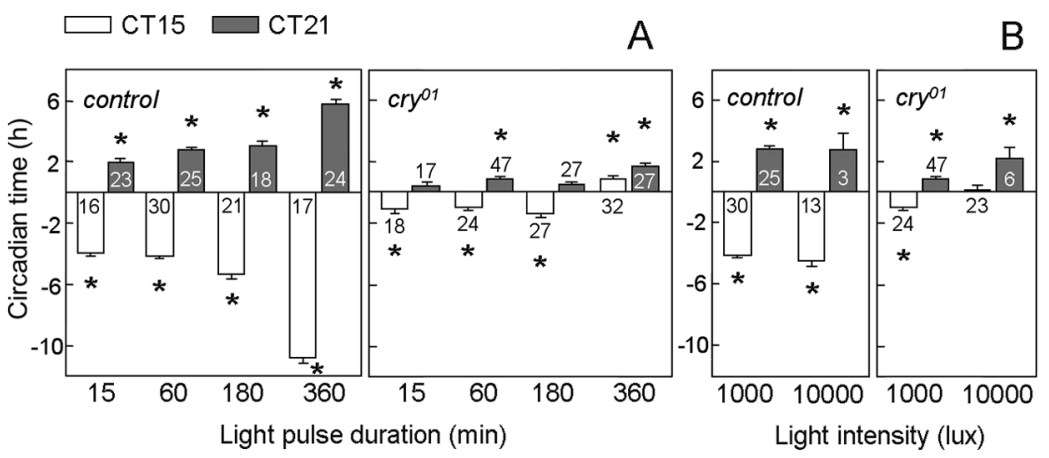

Figure 4. Phase shift responses to light pulses (1000 lux) of different duration (A) or intensity (B) applied either at CT15 or CT21 ( \pm SEM). (A) In control flies, the magnitude of advances and delays was clearly dependent on the duration of the light pulse (ANOVA for advances: $F_{3,86}=37.31, p<0.001$; ANOVA for delays: $F_{3,80}=159.92, p<$ 0.001). In $c r y^{01}$ mutants, the magnitude of advances and delays did not increase with increasing duration of the light pulses until 180 minutes (3 hours) (ANOVA for advances: $F_{2,81}=1.09, p=0.34$; ANOVA for delays: $\left.F_{2,66}=0.76, p=0.47\right)$. However, after 360-minute (6-hour) light pulses, a slight but significant increase of phase advances occurred at CT21 $(p=0.01)$, and the light pulses at CT15 resulted in phase advances instead of phase delays. (B) We light-pulsed 36 controls and $48 \mathrm{cry} y^{01}$ mutants with 10,000 lux at CT15 and 37 controls and $34 \mathrm{cry}{ }^{01}$ mutants at CT21. Surprisingly, the majority of flies became inactive after the light pulse, especially after the one administered at CT21. At CT21, the remaining 3 controls and 6 mutant flies phase-advanced their activity as expected. In control flies, the increase of light intensity to 10,000 lux did not change the magnitude of phase advances or delays (ANOVA for advances: $F_{1,26}=0.03$, $p=0.86$; ANOVA for delays: $F_{1,41}=2.21, p=0.14$ ). In $c r y^{01}$ mutants, the magnitude of phase delays was significantly affected by light intensity for CT15 pulses (ANOVA for advances: $F_{1,51}=2.28, p=0.14$; ANOVA for delays: $F_{1,45}=9.48, p=0.003$ ). The number of tested flies is indicated in, above, or below the columns, respectively, and phase shifts that were significantly different from unpulsed controls are marked by a star.

its shape during entrainment than after a long exposure to freerunning conditions (Mrosovsky, 1996; Johnson, 1999).

Our anchored PRC results for control flies are almost identical to the results of Dushay et al. (1990), although the latter authors used light pulses of 2000 lux and 10-minute duration and applied the light pulses on the fourth day of free-run. This indicates that the 2 methods to monitor a PRC yield very similar results in $D$. melanogaster. The magnitudes of phase shifts were also very similar to the other PRCs recorded for wild-type flies (Saunders et al., 1994; Emery et al., 1998; Rutila et al., 1998; Stanewsky et al., 1998; Suri et al., 1998): approximately 4 hours for phase delays and 1 to 3 hours for phase advances. This indicates that magnitudes depend little on the used light intensity ranging from 300 to 2000 lux and pulse duration from 10 minutes to 1 hour. The most likely explanation for this similarity is that the response to brief light pulses (up to 1 hour) was already

The magnitude of a phase shift with a 1-hour pulse is saturated for intensity in control flies but not in $c r y^{01}$ mutants, suggesting that the mutants have a low circadian light sensitivity.

\section{DISCUSSION}

PRCs are powerful tools to characterize the general properties as well as the light sensitivity of circadian clocks. There are 2 main ways to record a PRC. 1) The light pulse is applied while the oscillator is stably free-running in DD (Dushay et al., 1990; Saunders et al., 1994), or 2) the light pulse is applied in a freerun shortly after release from entraining conditions (also called anchored PRC) (Levine et al., 1994; Emery et al., 1998; Rutila et al., 1998; Stanewsky et al., 1998; Suri et al., 1998). We used the anchored PRC because this is the easiest method to light-pulse many flies at the same time and because the PRC shape soon after release from entrainment should be more reflective of saturated. This idea gets support from the present study, in which we could not increase phase shift magnitude of control flies at CT15 and CT21 by increasing irradiance to 10,000 lux. The saturation hypothesis is further supported by a seminal study of Nelson and Takahashi (1991), who tested the phaseshifting effects of brief light pulses ranging from 3 seconds to 1 hour in hamsters and found that 5-minute pulses evoked nearly the same response as 1-hour stimuli. They concluded that saturation had occurred after a light pulse duration of 5 minutes. Furthermore, the lowest number of photons was needed to reach saturation at this light pulse duration. In flies, the number of photons emitted during 1 hour at 10,000 lux seems to be far beyond saturation. The strong light had an unexpected additional effect on the activity of the flies because the majority of flies stopped running permanently, especially when the light pulse was administered at CT21. This is consistent with the activity-inhibiting effect of high intensity light we observed previously (Rieger et al., 2007). 
$c r y^{01}$ mutants also responded with significant phase shifts to 1-hour light pulses, although the magnitude of advances and delays was only about one quarter of the control flies. Shorter light pulses (15 minutes) only provoked significant phase delays, but not phase advances, indicating that $c r y^{01}$ mutants are already at the limit of their sensitivity. This is in accordance with a previous study that did not detect significant phase shifts in $c r y^{b}$ mutants to 10-minute light pulses of 1400 lux (Stanewsky et al., 1998). Without any doubt, cry mutants are much less light sensitive than wild-type flies. Nevertheless, the residual responses to light pulses (phase shifts of $\sim 1$ hour) can explain the rather normal entrainment of ${ }^{c r y}{ }^{01}$ mutants to LD cycles that was shown in many previous studies (Stanewsky et al., 1998; Emery et al., 2000b; Helfrich-Förster et al., 2001; Rieger et al., 2003; Bachleitner et al., 2007). Phase shift magnitudes of 1 hour appear very small, but they are not unusual for mammals in response to brief light pulses (see PRC atlas of Johnson [1990]). In fact, the re-entrainment properties of $\mathrm{cry}^{01}$ mutants (Fig. 2) closely resemble the ones reported for mammalian species (Aschoff et al., 1975).

In contrast to control flies, the light responses of $c r y^{01}$ mutants seemed not to be saturated in respect to irradiance: 1) the mutants significantly changed their phase-shifting behavior after increasing irradiance of the 1-hour light pulses from 1000 lux to 10,000 lux, and 2) they accelerated re-entrainment to an 8-hour phase delay of the LD cycle by almost 1 day when irradiance was increased to 10,000 lux.

In nature, brief light pulses rarely occur. Therefore, PRCs to brief pulses may fail to predict the behavior under LD 12:12 entrainment conditions. This is because longer exposure to light not only instantaneously phase-shifts the clock (nonparametric entrainment) but also influences its speed (parametric entrainment) (Aschoff, 1979; Wever, 1966). Thus, the application of longer light pulses can help to better understand entrainment. Comas et al. (2006) systematically monitored PRCs for single light pulses of different duration $(1,3,4,6,9,12$, and 18 hours) in mice. As expected, they found that longer light pulses caused a higher PRC amplitude, an effect that was also observed in other species including humans and flies (Gander and Lewis, 1983; Czeisler et al., 1989; Saunders et al., 1994). Here, we found that control flies increased phase delays to 11 hours (and phase advances to $\sim 6$ hours) when light pulse duration was extended to 6 hours, making understandable why fruit flies can entrain immediately to an 8-hour phase delay of the 12:12 LD cycle (Fig. 2). Comas et al. (2006) settled the strongest phase-shifting effect to the first half of the light pulse (the light action centered on average at $38 \%$ of the light pulse), possibly due to light adaptation of the circadian system and its photoreceptors. This might be also true for flies, at least for the controls.

The response of $c r y^{01}$ mutants to longer light pulses was fundamentally different from wild-type flies. No prominent increase in phase shift magnitude with increasing light pulse duration occurred in the mutants. Just when light pulse duration reached 6 hours, a small but significant increase of phase advances became evident. Therefore, the $c r y^{01}$ mutants are not so much disturbed in sensing light pulses than in collecting and integrating light input over time. The latter may be also reflected in the strange phase-shifting behavior of $c r y^{01}$ mutants after 6-hour light pulses at CT15. Instead of showing the expected delays, the flies exhibited phase advances (Fig. 4A). The reason for this behavior may lie in the fact that a 6-hour light pulse starting at CT15 will end at CT21, meaning that the end falls into the advance zone. Let us assume that $c r y^{01}$ mutants are not able to collect light properly over the 6 hours but instead sense mainly lights-on and lights-off. Then, very little phase shifts could be expected. If, for still unknown reasons, the light action is not centered on the first half of the light pulse but closer to lights-off, even small phase advances could result, and this is exactly what we observed. Nevertheless, this explanation can only partly explain the cry mutant results. We know already that cry mutants are not completely impaired in integrating light input over time. $\mathrm{cry}^{b}$ and $c r y^{01}$ mutants still show prominent period changes (parametric light effects) under LL (HelfrichFörster et al., 2001; Yoshii et al., 2004; Rieger et al., 2006; Dolezelova et al., 2007), indicating that an essential part of the parametric light input is mediated by the eyes and still intact in $c r y^{01}$ mutants. Most interestingly, constant light sensed via the eyes changed the velocity differently in different clock neurons, meaning that the molecular clock of some neurons ran faster and in other neurons slower under LL (Rieger et al., 2006). Perhaps 6-hour light pulses are long enough to elicit differential velocity changes in the different clock neurons and, as a consequence, caused the observed unusual phase shifts. Modeling the "circadian integrated response characteristic" (CIRC), as was recently suggested by Roenneberg 
et al. (2010), may help to explain the entrainment characteristics of CRY-less flies because this model makes no assumptions about how entrainment occurs (by phase shifts or velocity changes).

Leaving all speculation aside, there is one main difference between wild-type and CRY-deficient flies regarding parametric light effects: cry mutants do not become arrhythmic at LL, not even at high irradiances (Emery et al., 2000a; Helfrich-Förster et al., 2001; Yoshii et al., 2004; Rieger et al., 2006). In this respect, the clock of CRY-deficient flies appears similar to that of mammals because the clock of most mammalian species runs under constant dim light (Aschoff, 1979). On the molecular level, this difference is easy to understand because light-activated Drosophila CRY leads to degradation of TIM (Ceriani et al., 1999; Busza et al., 2004). After TIM has disappeared, PER cannot be stabilized, and as a consequence, the clock stops. Indeed, Saunders et al. (1994) noted that after 6-hour light pulses, the activity rhythm of wild-type flies always started with the same phase, suggesting that the clock had completely stopped and was restarted after lights-off. Mammalian-like CRY is not light sensitive, and thus, light will probably not completely stop the mammalian clock, at least not after light pulses of 6 hours. Only a longer light exposure will stop the clock, as recently reported in mice after a pulse longer than 15 hours (Chen et al., 2008).

The PRC for 12-hour light pulses shows that the clock of CRY-less flies is mainly light responsive at dawn and dusk. Such temporally restricted sensitivity must be sufficient for entrainment because dawn and dusk are the most important times at which a clock needs to respond to light (Bünning, 1969; Bachleitner et al., 2007). Because the light sensitivity of CRY-less flies is mediated by photoreceptor organs (as the compound eyes, the H-B eyelets, and possibly the ocelli), our results suggest that these organs transmit photic information to the clock only in the morning and evening. Thus, different photoreceptors may be responsible for the different parts of a PRC.

\section{ACKNOWLEDGMENTS}

The authors thank David Dolezel for the $c r y^{01}$ mutants and relevant control flies and Nicolai Peschel and Dirk Rieger for helpful discussions and comments on the article. This study was supported by the German Research Foundation (DFG; Fo207/11-3) and by the European Community (6th Framework Project EUCLOCK, no. 018741).

\section{CONFLICT OF INTEREST STATEMENT}

The author(s) have no potential conflicts of interest with respect to the research, authorship, and/or publication of this article.

\section{REFERENCES}

Aschoff J (1979) Circadian rhythms: influences of internal and external factors on the period measured in constant conditions. Z Tierpsychol 49:225-249.

Aschoff J, Hoffmann K, Pohl H, and Wever R (1975) Re-entrainment of circadian rhythms after phase-shifts of the zeitgeber. Chronobiologia 2:23-78.

Bachleitner W, Kempinger L, Wülbeck C, Rieger D, and Helfrich-Förster C (2007) Moonlight shifts the endogenous clock of Drosophila melanogaster. Proc Natl Acad Sci U S A 104:3538-3543.

Benito J, Houl JH, Roman GW, and Hardin PE (2008) The blue-light photoreceptor CRYPTOCHROME is expressed in a subset of circadian oscillator neurons in the Drosophila CNS. J Biol Rhythms 23:296-307.

Bünning E (1969) Die Bedeutung tagesperiodischer BlattbewegungenfürdiePräzisionderTageslängenmessung. Planta 86:209-217.

Busza A, Emery-Le M, Rosbash M, and Emery P (2004) Roles of the two Drosophila CRYPTOCHROME structural domains in circadian photoreception. Science 304:1503-1506.

Ceriani MF, Darlington TK, Staknis D, Mas P, Petti AA, Weitz CJ, and Kay SA (1999) Light-dependent sequestration of TIMELESS by CRYPTOCHROME. Science 285:553-556.

Chen R, Seo D, Bell E, von Gall C, and Lee C (2008) Strong resetting of the mammalian clock by constant light followed by constant darkness. J Neurosci 28:11839-11847.

Comas M, Beersma DGM, Spoelstra K, and Daan S (2006) Phase and period responses of the circadian system of mice (Mus musculus) to light stimuli of different duration. J Biol Rhythms 21:362-372.

Czeisler CA, Kronauer RE, Allan JS, Duffy JF, Jewett ME, Brown EN, and Ronda JM (1989) Bright light induction of strong (type 0 ) resetting of the human circadian pacemaker. Science 244:1328-1333.

Dolezelova E, Dolezel D, and Hall JC (2007) Rhythm defects caused by newly engineered null mutations in Drosophila's cryptochrome gene. Genetics 177:329-345.

Dushay MS, Konopka RJ, Orr D, Greenacre ML, Kyriacou CP, Rosbash M, and Hall JC (1990) Phenotypic and genetic analysis of Clock, a new circadian rhythm mutant in Drosophila melanogaster. Genetics 125:557-578.

Emery P, So WV, Kaneko M, Hall JC, and Rosbash M (1998) CRY, a Drosophila clock and light-regulated cryptochrome, is a major contributor to circadian rhythm resetting and photosensitivity. Cell 95:669-679.

Emery P, Stanewsky R, Hall JC, and Rosbash M (2000a) A unique circadian-rhythm photoreceptor. Nature 404:456-457. 
Emery P, Stanewsky R, Helfrich-Förster C, Emery-Le M, Hall JC, and Rosbash M (2000b) Drosophila CRY is a deep brain circadian photoreceptor. Neuron 26:493-504.

Gander PH and Lewis RD (1983) Phase-resetting action of light on the circadian activity rhythm of Rattus exulans. Am J Physiol 245:R10-R17.

Glaser WR (1978) Varianzanalyse. Stuttgart: Gustav Fischer Verlag.

Helfrich-Förster C (1998) Robust circadian rhythmicity of Drosophila melanogaster requires the presence of lateral neurons: a brain-behavioral study of disconnected mutants. J Comp Physiol A 182:435-453.

Helfrich-Förster C, Winter C, Hofbauer A, Hall JC, and Stanewsky R (2001) The circadian clock of fruit flies is blind after elimination of all known photoreceptors. Neuron 30:249-261.

Hirsh J, Riemensperger T, Coulom H, Iche M, Coupar J, and Birman S (2010) Roles of dopamine in circadian rhythmicity and extreme light sensitivity of circadian entrainment. Curr Biol 20:209-214.

Johnson CH (1990) An Atlas of Phase Responses Curves for Circadian and Circatidal Rhythms. Nashville: Department of Biology, Vanderbilt University. p 715.

Johnson CH (1999) Forty years of PRCs: what have we learned? Chronobiol Int 16:711-743.

Johnson CH (1992) Phase response curves: what can they tell us about circadian clocks? In Circadian Clocks from Cell to Human, Hiroshige T and Honma K, eds, pp 209-246. Sapporo: Hokkaido University Press.

Konopka RJ, Pittendrigh C, and Orr D (1989) Reciprocal behaviour associated with altered homeostasis and photosensitivity of Drosophila clock mutants. J Neurogenet $6: 1-10$.

Levine JD, Casey CL, Kalderon DD, and Jackson FR (1994) Altered circadian pacemaker functions and cyclic AMP rhythms in the Drosophila learning mutant dunce. Neuron 13:967-974.

Morin LP and Allen CN (2006) The circadian visual system. Brain Res Rev 51:1-60.

Mrosovsky N (1996) Methods of measuring phase shifts: why I continue to use an Aschoff type II procedure despite the skepticism of referees. Chronobiol Int 13:387-392.

Nelson DE and Takahashi JS (1991) Sensitivity and integration in a visual pathway for circadian entrainment in the hamster (Mesocricetus auratus). J Physiol 439:115-145.

Ohata K, Nishiyama H, and Tsukahara Y (1998) Action spectrum of the circadian clock photoreceptor in Drosophila melanogaster. In Biological Clocks: Mechanisms and Applications, Touitou Y, ed, pp 167-171. Amsterdam: Elsevier.

Peschel N, Veleri S, and Stanewsky R (2006) Veela defines a molecular link between Cryptochrome and Timeless in the light-input pathway to Drosophila's circadian clock. Proc Natl Acad Sci U S A 103:17313-17318.
Rieger D, Fraunholz C, Popp J, Bichler D, Dittmann R, and Helfrich-Förster C (2007) The fruit fly Drosophila melanogaster favors dim light and times its activity peaks to early dawn and late dusk. J Biol Rhythms 22:387-399.

Rieger D, Shafer OT, Tomioka K, and Helfrich-Förster C (2006) Functional analysis of circadian pacemaker neurons in Drosophila melanogaster. J Neurosci 26:2531-2543.

Rieger D, Stanewsky R, and Helfrich-Förster C (2003) Cryptochrome, compound eyes, Hofbauer-Buchner eyelets, and ocelli play different roles in the entrainment and masking pathway of the locomotor activity rhythm in the fruit fly Drosophila melanogaster. J Biol Rhythms 18:377-391.

Roenneberg T, Remi J, and Merrow M (2010) Modeling a circadian surface. J Biol Rhythms 25:340-349.

Rutila JE, Maltseva O, and Rosbash M (1998) The timSL mutant affects a restricted portion of the Drosophila melanogaster circadian cycle. J Biol Rhythms 13:380-392.

Saunders DS, Gillanders SW, and Lewis RD (1994) Lightpulse phase response curves for the locomotor activity rhythm in Period mutants of Drosophila melanogaster. J Insect Physiol 40:957-968.

Stanewsky R, Kaneko M, Emery P, Beretta B, Wager-Smith K, Kay SA, Rosbash M, and Hall JC (1998) The cry mutation identifies cryptochrome as a circadian photoreceptor in Drosophila. Cell 95:681-692.

Suri V, Qian Z, Hall JC, and Rosbash M (1998) Evidence that the TIM light response is relevant to light-induced phase shifts in Drosophila melanogaster. Neuron 21: 225-234.

Veleri S, Brandes C, Helfrich-Förster C, Hall JC, and Stanewsky R (2003) A self-sustaining, light-entrainable circadian oscillator in the Drosophila brain. Curr Biol 13:1758-1767.

Veleri S, Rieger R, Helfrich-Förster C, and Stanewsky R (2007) Hofbauer-Buchner eyelet affects circadian photosensitivity and coordinates TIM and PER expression in Drosophila clock neurons. J Biol Rhythms 22:29-42.

Wegener C, Hamasaka Y, and Nässel DR (2004) Acetylcholine increases intracellular $\mathrm{Ca}^{2+}$ via nicotinic receptors in cultured PDF-containing clock neurons of Drosophila. J Neurophysiol 91:912-923.

Wever RA (1966) Ein mathematisches Modell für die circadiane Periodik. Zeitschrift für angewandte Mathematik und Mechanik 46:148-157.

Yoshii T, Funada Y, Ibuki-Ishibashi T, Matsumoto A, Tanimura T, and Tomioka K (2004) Drosophila cry ${ }^{b}$ mutation reveals two circadian clocks that drive locomotor rhythm and have different responsiveness to light. J Insect Physiol 50:479-488.

Yoshii T, Todo T, Wülbeck C, Stanewsky R, and HelfrichFörster C (2008) Cryptochrome is present in the compound eyes and a subset of Drosophila's clock neurons. J Comp Neurol 508:952-966. 